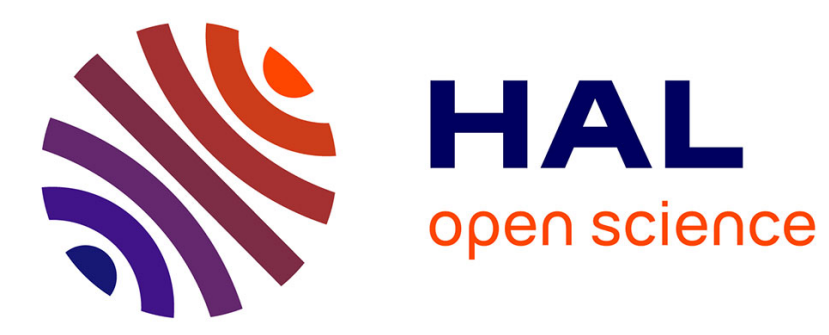

\title{
Le documentaire élargi au web
}

Evelyne Broudoux

\section{To cite this version:}

Evelyne Broudoux. Le documentaire élargi au web. Les Enjeux de l'information et de la communication, 2011, pp.3. 10.3917/enic.hs03.0003 . sic_00715835

\section{HAL Id: sic_00715835 \\ https://archivesic.ccsd.cnrs.fr/sic_00715835}

Submitted on 9 Jul 2012

HAL is a multi-disciplinary open access archive for the deposit and dissemination of scientific research documents, whether they are published or not. The documents may come from teaching and research institutions in France or abroad, or from public or private research centers.
L'archive ouverte pluridisciplinaire HAL, est destinée au dépôt et à la diffusion de documents scientifiques de niveau recherche, publiés ou non, émanant des établissements d'enseignement et de recherche français ou étrangers, des laboratoires publics ou privés. 
Pre-print pour « Les Enjeux de l'information et de la communication », 2011.

\title{
Le documentaire élargi au web
}

\author{
Evelyne Broudoux
}

\section{INTRODUCTION}

Sur le web, la panoplie des genres éditoriaux s'élargit progressivement : blogs, portails, wikis collaboratifs, webzines, vidéos «citations », etc. Depuis 2004, nous assistons à l'émergence d'un nouveau type de création - le web-documentaire ${ }^{1}$ - qui allie films documentaires et spécificités du web et dont les techniques d'écriture apparaissent se stabiliser. Des organismes comme le Centre National du Cinéma et de l'image animée (CNC) n'hésitent pas à subventionner ce type de production et des médias comme Arte ou Le Monde montent des plateformes dédiées à ce nouveau mode d'expression.

Qu'appelle-t-on exactement web-documentaire? Un agencement spécifique scénarisant la consultation sur le web d'un documentaire thématique sous une forme participative. On peut le définir comme :

- un documentaire réalisé en vidéos, en bandes sons, en textes et en images,

- dont la scénarisation tient compte de l'interactivité

- dans la fragmentation des récits

$\circ$ et dans l'interface graphique

- et qui s'insère dans un dispositif personnalisant la communication avec l'internaute (réseaux sociaux, commentaires, etc.).

Il existe une multiplicité des appellations comme animations interactives, petites œuvres multimédia (POM), documentaires multimédias, cyberdocumentaires, qui indique l'émergence d'un nouveau style qui n'a pas encore trouvé son nom et qui oscille entre plusieurs directions de développement.

\section{LA TRANSFORMATION DES MEDIAS PAR LE NUMERIQUE}

\section{Changements dans le mode de consommation des médias}

Dans son livre «Convergence culture », Henry Jenkins tente de décrire cette phase de turbulences dans laquelle sont plongés les médias de diffusion qui opèrent dans un environnement devenu réactif et participatif et qui doivent coopérer avec une audience moins fidélisable, c'est-à-dire qui est capable de migrer pour réaliser des expériences qui lui conviennent. Il nomme convergence l'intersection entre au moins trois processus : celui de la mise en flux des contenus, celui de la multiplicité des plateformes médiatiques et celui du caractère versatile de l'audience.

\footnotetext{
${ }^{1}$ « Le webdocumentaire (souvent raccourci en webdoc ou webdocu) est un média interactif d'information éditorialisé présent sur le web, avec une interface regroupant du Richmedia. À un reportage vidéo principal s'ajoute une série de liens qui permettent d'enrichir l'information avec des vidéos, textes, photos, sons complémentaires, réseaux sociaux/commentaires des internautes... » in : FreeLens : Pour une photographie d'utilité publique. POM, vidéographie, webdocumentaire... Concrètement, qu'est-ce que c'est ? [En ligne]_http://www.freelens.fr/pomvideographie-webdocumentaire-definition/ (consultée le 5/12/2010)
} 
L'arrivée des web-documentaires s'insère dans un paysage médiatique caractérisé par des changements dans le mode de consommation des médias. Alors que le lectorat de la presse écrite continue de baisser inexorablement, la télévision a progressivement cédé sa première place à l'internet en termes de temps passé devant l'appareil récepteur chez les «digital natives». Deux remarques s'ensuivent :

- La récente et forte croissance du visionnage de vidéos sur internet laisse augurer l'essor d'un marché de masse constitué par un gros volume de vidéos professionnelles souvent revisitées par des amateurs et s'adressant à une large audience sur les web TV (ex : Current.tv) et les plate-formes (ex: Dailymotion, YouTube) et se relayant sur de multiples réseaux sociaux. Ces vidéos étant rarement monétisées, leurs revenus publicitaires générés sont basés sur la mesure quantitative de l'audience qui les visionne.

- Dans le déluge informationnel, la captation de l'attention passe par des artifices interactifs maintenant l'internaute en immersion dans des univers contraints et addictifs (réseaux sociaux, etc.) fortement inspirés par l'univers des jeux vidéo. Depuis les simulateurs de vols pour l'apprentissage du pilotage des avions à l'utilisation de « jeux sérieux » (ex : pour la simulation d'exercices militaires), les systèmes de représentation du réel n'ont cessé de se diversifier. Avec la notion de « réalité augmentée » sur mobiles, une hybridation est atteinte, celle de l'image captée en temps réel sur laquelle viennent s'inscrire différentes formes d'information (infographies, logos, publicités, données, etc.) documentant les contenus captés.

La télévision connectée c'est-à-dire raccordée directement ou indirectement à l'internet est en passe de remplacer la télévision hertzienne. Conçue dès les débuts du web, la «télévision interactive » intéresse aujourd'hui de près les industriels (opérateurs réseaux, télécommunication audiovisuelle, fabricants d'écrans, de récepteurs) et les acteurs des industries de la culture ; le visionnage actuel massif des vidéos prouvant que l'offre aura du succès. Composée d'un appareil récepteur-émetteur et d'un écran multifenêtres et tactile, elle devrait proposer des contenus délinéarisés aux côtés du flux télévisuel habituel autorisant le visionnage de vidéos type YouTube/Dailymotion, la TV de rattrapage (catchTV, ex : Hulu, arte+7) et les vidéos à la demande (Vod, ex : Netflix, mySkreen), mais aussi l'usage de services informatiques susceptibles de la transformer en véritable "poste de commande » domotique. L'intérêt pour les annonceurs publicitaires d'une télévision connectée est d'avoir accès à des systèmes de mesure de l'interactivité de l'audience impossibles à imaginer dans le cas d'une télévision hertzienne.

\section{Contexte économique de l'apparition des web-documentaires}

En économie des médias, les web-documentaires font partie des «biens informationnels » qui concentrent de l'information librement appropriable (ex : connaissances, mélodies) et il s'agit également de «biens publics » régis par un principe de «non rivalité » dans la mesure où leur usage n'induit pas une diminution de leur quantité pour d'autres consommateurs. [Chantepie, Le Diberder, 2010] constatent un mouvement dans les modèles économiques traditionnels des diffuseurs et des éditeurs. Les contenus numériques fixés sur les supports physiques lorsqu'ils sont immatérialisés sur les réseaux changent de statut : de biens privés susceptibles de paiement direct, ils passent à l'économie des biens collectifs et deviennent non-rivaux. Ce qui engendre toute une série de changements pour les ayants-droits dont les revenus cessent d'être proportionnels mais vont dépendre d'allocations réglementées, mais aussi pour les bénéficiaires consommateurs transformés en «passagers clandestins » qui voient leurs pratiques (écoute musicale, lecture d'e-books) rendues illégales. Il ne faudrait pas oublier non plus les conséquences de 
développement pour les industries techniques qui bénéficient des effets d'externalité positive (DRM, etc.).

Subventionnés en amont par le CNC qui soutient l'expérimentation de nouvelles formes d'expression, les documentaires portés sur le web sont bénéficiaires de ce changement de régime. Mais ils servent aussi de tests à des médias qui cherchent à diversifier leur production et tentent de trouver de nouveaux modèles économiques pour leurs futurs revenus. C'est ainsi que des éditeurs de presse écrite et audiovisuelle comme Le Monde, Arte, France 5, France 24, Canal+ se sont lancés dans la publication régulière de «web-documentaires » dont la particularité est d'avoir été conçus pour être consultés sur le web. Cette orientation de mise en ligne de contenus « interactifs » de la part d'acteurs de la presse audiovisuelle et imprimée pourrait être envisagée comme un rodage de nouvelles techniques d'écriture adaptées au web.

\section{PROBLEMATIQUES DU WEB-DOCUMENTAIRE}

\section{Une pluralité des profils chez les créateurs de web-documentaires}

Le web-documentaire apparait comme le symbole d'un renouveau de la production créative à la croisée des chemins documentaires et journalistiques. Il s'agit d'un format d'expression qui s'autonomise par rapport aux anciens supports. Il bénéficie d'une relative autonomie dans la recherche de ses marques énonciatives sur le web. Informer par l'intermédiaire d'un web-documentaire c'est construire une forme qui prend en compte les caractéristiques communicationnelles du web tout en poursuivant des objectifs d'intérêt général.

Il existe une grande pluralité d'acteurs investis dans les web-documentaires. Les créateurs sont des vidéastes et/ou photographes, rédacteurs et/ou preneurs de son, journalistes reporters d'images (jri) et/ou scénaristes, etc. Deux grands profils se côtoient : photographes et documentaristes issus du cinéma se situant dans une démarche d'auteur et journalistes professionnels. Ne bénéficiant pas des mêmes régimes juridiques, des ambivalences se créent, par exemple lorsque des web-documentaristes se voient refuser l'attribution d'une carte de presse sous prétexte qu'ils étaient réalisateurs ou auteurs, alors que les contenus produits étaient réalisés à partir d'enquêtes journalistiques de terrain.

Les web-documentaires possèdent leurs circuits de financement, de promotion, leurs concours ${ }^{2}$ et récompenses, leurs portails ${ }^{3}$ et spécialistes blogueurs ${ }^{4}$, leurs plateformes labos ${ }^{5}$ et leurs nouvelles agences dédiées. Nombreux parmi les acteurs sont ceux qui reconnaissent une difficulté à financer le portage sur le web de documentaires conçus pour l'audiovisuel; des coûts distincts entrent pour la production audiovisuelle ou photographique et la post-production. Des sociétés productrices de web-documentaires se sont donc montées comme Upian, Narrative ou Honkytonk qui a ainsi développé son propre logiciel de «montage non-linéaire » Klynt, permettant «de diviser par trois les coûts du développement Web de chaque création, coûts qui représentent, en général, 30 à $40 \%$ du budget global ${ }^{6}$ de chaque webdocumentaire. Mais d'autres applications conçues de manière à faciliter le montage en ligne comme 3WDOC de l'agence Hecube sont susceptibles d'ouvrir le genre à l'autoproduction et aux amateurs.

\footnotetext{
${ }^{2}$ FRANCE 24 et RFI organisent le Prix du Webdocumentaire qui s'inscrit pour sa troisième édition en 2011 dans le cadre de Visa pour l'Image à Perpignan, le plus important festival international de photojournalisme. [En ligne] http://www.france24.com/fr/FRANCE-24-RFI-prix-du-webdocumentaire-2011 (consultée le 22 juillet 2011).

${ }^{3}$ Webdocu [En ligne] http://webdocu.fr/web-documentaire/ (consultée le 22 juillet 2011).

${ }^{4}$ Interactive Documentary [En ligne] http://www.interactivedocumentary.net/ (consultée le 22 juillet 2011).

${ }^{5}$ I-docs [En ligne] http://www.i-docs.fr/ (consultée le 22 juillet 2011).

${ }^{6}$ http://numerico.wordpress.com/2010/04/09/klynt-la-solution-pour-la-creation-sur-internet/ (consultée le 5/12/2010)
} 
Les objectifs des documentaires audiovisuels à caractère cinématographique sont souvent de questionner des préoccupations sociétales, des modes de vie, des faits sociaux. Il se démarque de la fiction par sa prétention à capter des morceaux de réalité appartenant à un contexte actuel ou reconstitué sans passer par l'invention d'une histoire dont les comédiens interprètent le scénario dans une mise en scène. On peut dire que la principale fonction du documentaire est de traduire le réel :

- en énonçant un point de vue d'auteur sur une réalité perçue,

- en créant une distance narrative entre les faits représentés et le spectateur placé dans une situation de témoin,

- en sollicitant une prise de conscience active chez le spectateur qui doit identifier ce point de vue et l'interpréter.

Cette traduction du réel qui s'opère avec les techniques appartenant à la narrativité cinématographique engendre une reconstruction proche de la problématique fictionnelle : les fonctionnalités de la prise d'images et leur esthétique, l'indispensable scénarisation et les opérations de montage, la transformation de personnes en personnages, etc.

Il y a près de vingt ans, Jean-Paul Colleyn jugeait illusoire l'établissement d' " une définition formelle globale et concise » du documentaire face à l'éclatement de «l'ensemble de la production en sous-genres tels que fiction documentée, documentaire de création, docu-drame, documentaire fictionnalisé » mais maintenait cependant que la distinction entre « œuvre inventée » et « réalité réinventée » par le cinéma était une nécessité pour sauvegarder l'identité du genre documentaire.

Aussi, dans cette optique, l'utilisation par les web-documentaires d'artifices narratifs pour représenter le réel vient s'inscrire dans la continuité du genre. Cependant, nous émettons l'hypothèse que si l'interactivité enrichit les espaces narratifs des web-documentaires, elle risque aussi d'abolir la distance nécessaire à la réflexion. Pour cela nous nous proposons de vérifier comment la narratologie alimente les espaces documentaires et vice-versa pour ce type de production dans un corpus de web-documentaires.

Ces vingt dernières années auront vu éclater les frontières des genres traditionnels basés sur la séparation des récits fictionnels de ceux narrant des faits réels. Les œuvres de fiction comme les romans ou les films de cinéma se sont vues concurrencées par de multiples formes de récits comme les autofictions, les jeux en réalité alternée, les «reality show » et toutes sortes de reconstructions imaginées de faits divers. Informer n'est plus collecter des événements à destination d'un public exerçant un esprit critique, c'est aussi construire des formes destinées à manipuler les idées et les esprits dans des objectifs divers qui vont de la distraction de la réalité à l'engagement citoyen. Du «storytelling » politique aux jeux « sérieux » employés pour l'adhésion à une cause (cf. l'exemple récent d'advocacy pour le Darfour ${ }^{7}$ ), le récit multisupports s'est enrichi de nouvelles définitions correspondant à de nouveaux produits de communication basés sur la narration: cross media lorsqu'il y a duplication ou adaptation (ex : Thealexanderwilsonproject) et trans media, lorsque la conception de l'univers narratologique englobe des points d'entrée différents dans l'histoire selon le support utilisé (ex : Panzerchocolate ${ }^{8}$ ). Il faut cependant noter que la différence entre ces deux genres apparaît souvent ténue.

\footnotetext{
${ }^{7}$ Onthegroundreporter : http://onthegroundreporter.com/

${ }^{8}$ Panzerchocolate : http://www.filmutea.com/panzerchocolate
} 


\section{La grille d'analyse adoptée}

Nous observerons ici les parcours de lecture réalisés par les agencements spécifiques des webdocumentaires articulant des récits et les multiples formes de la matière documentaire.

Nous étudions la présentation du récit principal, des analyses, des témoignages, paroles d'experts, d'amateurs et de professionnels en même temps que les différentes combinaisons des espaces narratifs et documentaires. Nous nous appuyons sur la théorie du récit différenciant récit/histoire/narration. Rappelons qu'un récit est une histoire (constituée de faits réels interprétés ou inventés) racontée par quelqu'un (personnage, présentateur, voix off, etc.). Gérard Genette distingue le récit (signifiant, le discours ou le texte ou l'image) de l'histoire (signifié, le contenu, les événements racontés) et de la narration (énonciation, ordre, durée, points de vues).

\section{DES CONVENTIONS D'ECRITURE DECELABLES}

\section{La ligne temporelle}

La ligne temporelle (timeline) comme élément structurant dominant de la présentation multimédia révèle la volonté de préserver le fil conducteur de la narration principale. Elle constitue une approche choisie par maints auteurs peut-être également à cause de l'économie de moyens qu'elle permet en termes de post-production. Véritable colonne vertébrale du web-documentaire, elle articule récits et éléments complémentaires de documentation comme sur l'exemple de QUATRO HORAS (France 5) qui présente une ville construite par des mineurs, chercheurs d'or au Pérou, ayant réussi à obtenir le droit d'exploiter en coopérative la mine et gagner ainsi en légitimité.

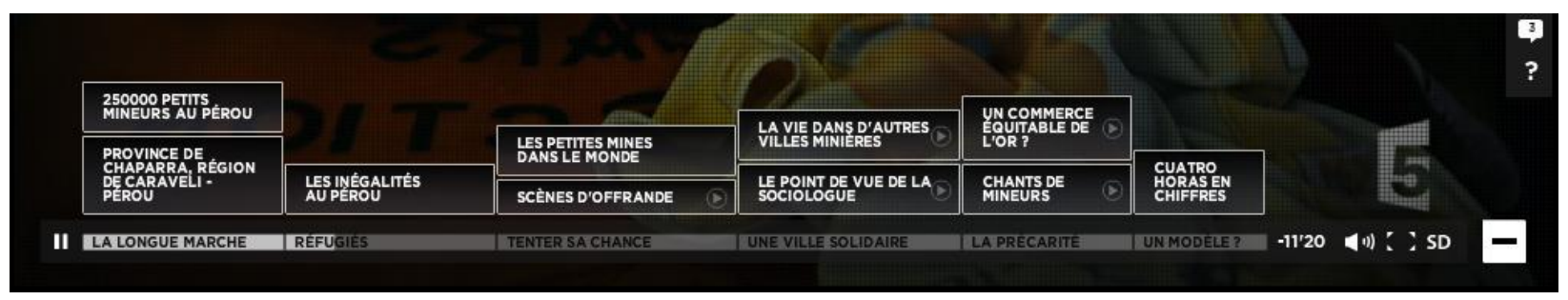

Figure 1 - Affichage de l'ensemble des éléments de documentation complémentaires accessibles au fur et à mesure de la progression du récit sur sa ligne temporelle. L'espace documentaire s'affiche sur l'espace narratif. 


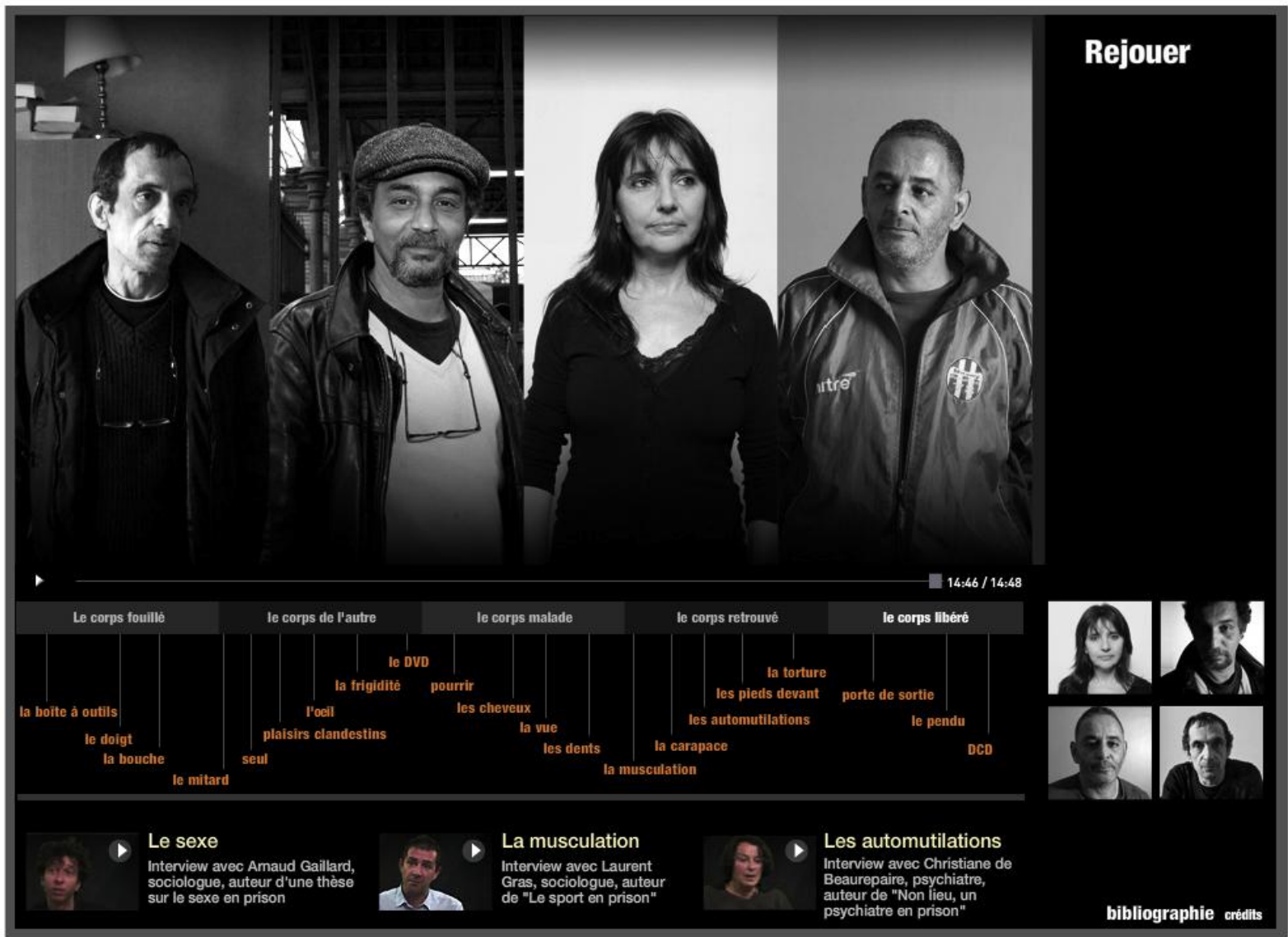

Figure 2 - Voix de détenus et voix d'experts contribuent ensemble à apporter un éclairage sur la situation du corps en prison. Il n'existe pas ici de véritable séparation entre espaces narratifs et espaces documentaires ; ce qui fait la séparation, c'est la différence de statut des voix.

Dans LE CORPS INCARCÉRÉ (Le Monde), le récit principal se charge au lancement de la page web et démarre automatiquement avec des éléments sonores métaphoriques forts (claquement d'une porte de couloir, bruits de pas, etc.). La structuration de la ligne temporelle est réalisée en cinq parties qui se composent elles-mêmes de séquences thématiques titrées. Une fois la totalité du diaporama sonore chargé, chaque séquence peut être revue et réécoutée en indépendance des autres.

Dans ce web-documentaire, la séparation des témoignages des détenus et l'analyse des experts est réalisée par l'interface graphique. A droite du récit principal se trouvent les photos des quatre détenus interviewés qui donnent accès à des éléments biographiques. La partie inférieure de l'écran comporte la parole de trois experts, une partie bibliographie et les crédits. 


\section{Le récit à embranchements multiples}

iROCK (Orange) propose à l'internaute de « déambuler » dans les coulisses d'un grand festival rock : les Eurockéennes de Belfort (2009). L'internaute est amené à suivre des journalistes, des musiciens et d'autres personnages sur quatre parcours d'une durée d'environ quinze minutes, menant vers quatre-vingt dix séquences vidéo de une à trois minutes chacune prenant sur le vif la vie quotidienne des professionnels sur un festival. Chaque séquence est légendée et à leur fin, deux propositions sont faites (voir figure 3).

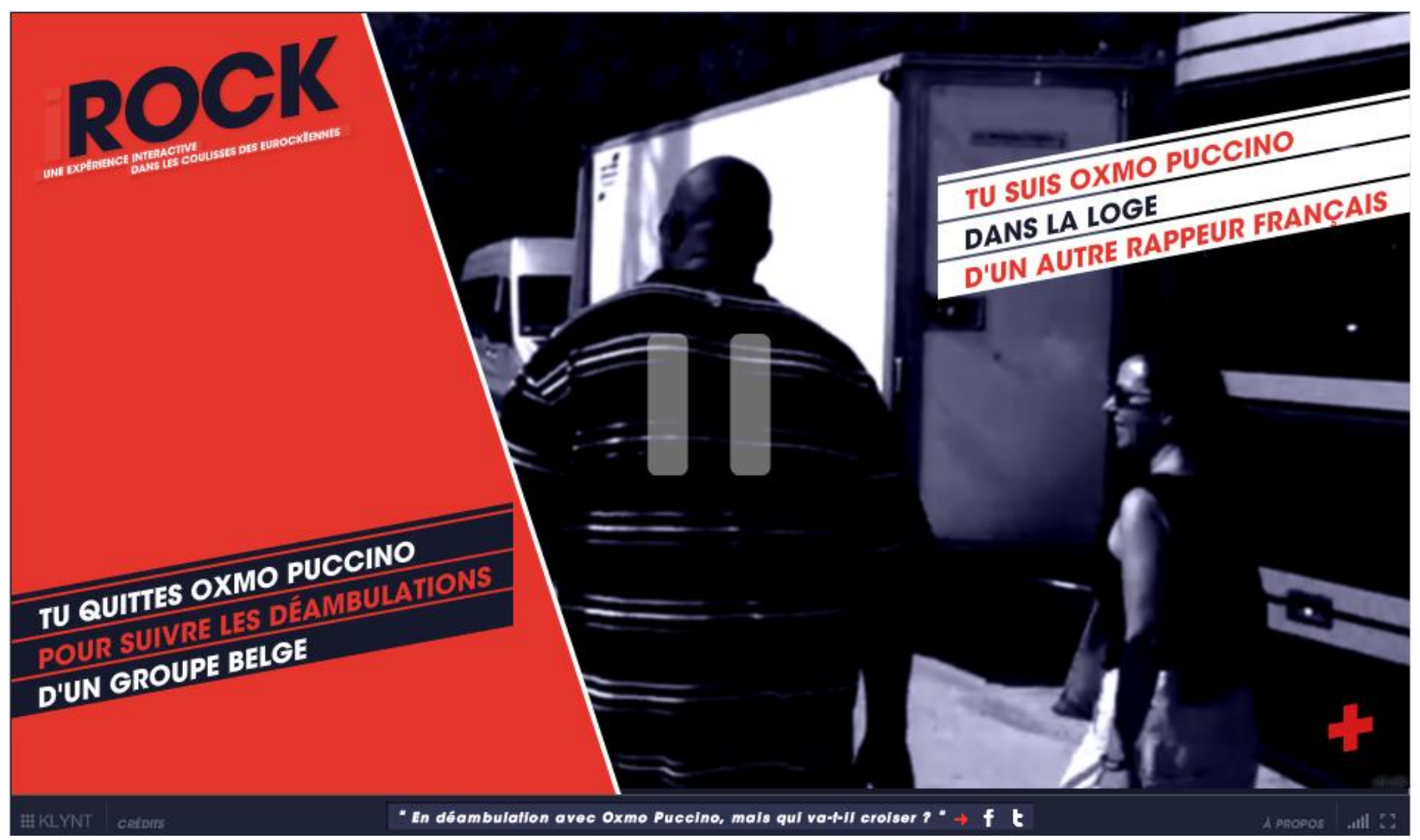

Figure 3 - Bifurcation dans l'espace narratif qui s'efface totalement pour laisser la place à l'espace documentaire lorsqu'il est convoqué (signe + ).

L'originalité du dispositif tient à la possibilité d'accéder à des fiches multimédia par artiste qui se composent de brèves biographies, discographies, extraits filmés de concerts à visionner sur Dailymotion et téléchargement en mp3 sur Orange) et autres liens web menant vers les sites des artistes. L'appel au référencement des séquences sur les réseaux sociaux est constant.

Le supplément documentaire qui vient compléter la balade cinématographique a une visée clairement promotionnelle : de la légende de chaque séquence qui affiche «partage cette séquence » au passage de la souris sur différents réseaux sociaux à l'entourage du web-documentaire incitant à visionner d'autres vidéos sur les mêmes thèmes.

LE CHALLENGE (Canal+) se présente comme une enquête interactive sur un grand procès lié à la dégradation environnementale et mené contre la société Texaco par les indiens de la forêt amazonienne. Tiré d'un film TV de 52 minutes, le web-documentaire d'une durée d'environ vingt minutes, repose sur la forte implication de l'internaute, scénarisée et intégrée dès la prise d'images. 
Comme pour IRock, l'internaute a le choix entre deux possibilités dans l'enchainement des séquences, mais il s'agit soit de continuer l'enquête en écoutant les personnages répondre aux questions choisies, soit de permuter d'espace et d'accéder à l'info préparatoire aux entretiens.

Dans Le Challenge, l'espace documentaire se matérialise par un bloc-notes qui donne accès à la matière permettant de contextualiser le récit principal filmé. Divisé en deux parties, il accueille sur le côté droit une carte zoomable de l'équateur sur laquelle sont localisées la position de l'internaute et les différentes séquences à visionner. Sur le côté gauche, l'internaute trouve à sa disposition le matériel documentaire mettant en perspective le procès dans lequel il se trouve investi : des caractéristiques chiffrées de l'équateur en passant par une explication politique de la colonisation et des vidéos historiques, tout est classé en cinq catégories (équateur, le procès, pétrole, le yasun, annexes). La consultation de son blocnotes est scénarisée et est incluse dans les séquences vidéos du récit principal (par exemple pour préparer ses interviews).

Dans L'OBÉSITE EST-ELLE UNE FATALITÉ? (France 5), l'internaute est présenté comme un «protagoniste» du web-documentaire. Invité à mener une enquête sur le caractère épidémique de l'obésité, il doit prendre des décisions et choisit des questions à poser (sous forme de légendes). Des extraits de vidéos y répondent ou des enregistrements audio sur des photos. Le découpage de séquences d'entretiens et de mini-reportages peut être cependant visionné de manière totalement linéaire. Alors que dans les deux web-documentaires précédents, l'espace documentaire masque complètement l'espace narratif, dans celui-ci il se superpose en un écran plus petit sur la séquence vidéo en cours.

\section{L'interface graphique métaphorique}

BREVES DE TROTTOIR est un web-documentaire autoproduit de Marc Lustigman et Noam Roubah, de la conception à la publication web, dans lequel l'activité de consultation des interviews est totalement distincte de la navigation de l'interface qui est elle-même porteuse d'une énonciation piétonnière. Fortement métaphorique, l'interface se compose de murs décrépis bordant une rue sur laquelle se trouvent différents éléments symboliques de la vie parisienne: plan de Paris, kiosque à journaux, pancarte «Défense d'afficher », plaque de rue, etc. Contrairement aux pages web traditionnelles, la navigation se déroule de droite à gauche et non de haut en bas, tout au long de la rue qui accueille les différentes figures interviewées. Chaque témoignage prend place dans une télévision des années soixante autour de laquelle sont disposés les «suppléments » documentaires : images, textes, sons qui s'incarnent sur leur support représentés (photos, lettres, poste de radio). 


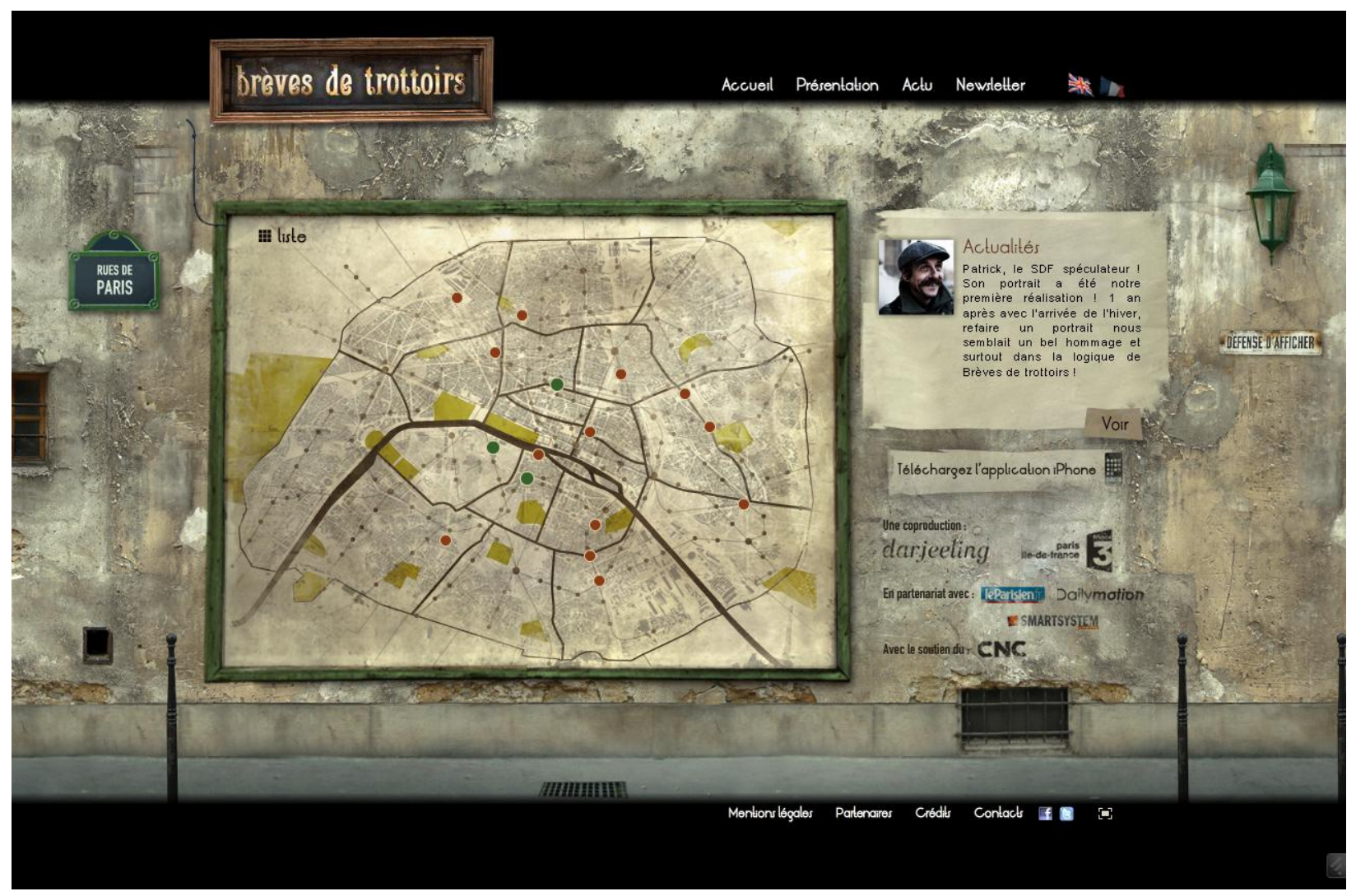

Figure 4 - La rue et le plan de Paris, métaphores d'accès aux portraits de parisien(ne)s.

Dans Brèves de trottoir, il n'existe pas de partie documentaire se différenciant de la partie captée en sons, images et vidéos. Chaque portrait se divise en un entretien principal, réalisé en bande son-images défilantes ou vidéos, accompagné de «bonus », nommés ainsi par les auteurs, qui sont des suppléments informationnels concernant uniquement la vie personnelle des personnages. En réalité, la contextualisation est procurée par l'interface bourrée de métaphores qui pose des Parisiens aux vies un peu marginales, aux occupations originales, dans des mondes qui leur appartiennent. Il s'agit donc d'un web-documentaire qui témoigne plus qu'il ne décrit.

La superproduction PRISON VALLEY (Arte.tv) est aussi réalisée sur le registre de la séparation des activités de consultation des espaces narratifs et documentaires par l'interface graphique. Toute la scénarisation de l'activité de l'internaute au sein du web-documentaire part de la chambre d'un Motel et ses métaphores de l'activité d'enquête (calepin sur la commode, souvenirs et documents qui s'accumulent sur le lit au fur et à mesure de l'avancée dans le documentaire, etc.). Après avoir visionné la première séquence de ce qui se présente comme un « roadmovie », l'internaute est plongé dans « sa » chambre d'où il accède à la matière documentaire qui éclaire et contextualise les séquences vidéos qu'il visionne les unes après les autres dans l'ordre imposé par les auteurs. C'est un petit peu la spécificité de Prison Valley que de ne pas briser la structure narrative des séquences vidéos ou d'assemblages sons-images. Il n'existe aucun moyen pour l'internaute de manquer une séquence principale et s'il le tente, il est ramené à la première séquence et obligé de repasser par la case départ. Ce parcours fortement contraint est aussi représentatif de l'univers carcéral décrit. 


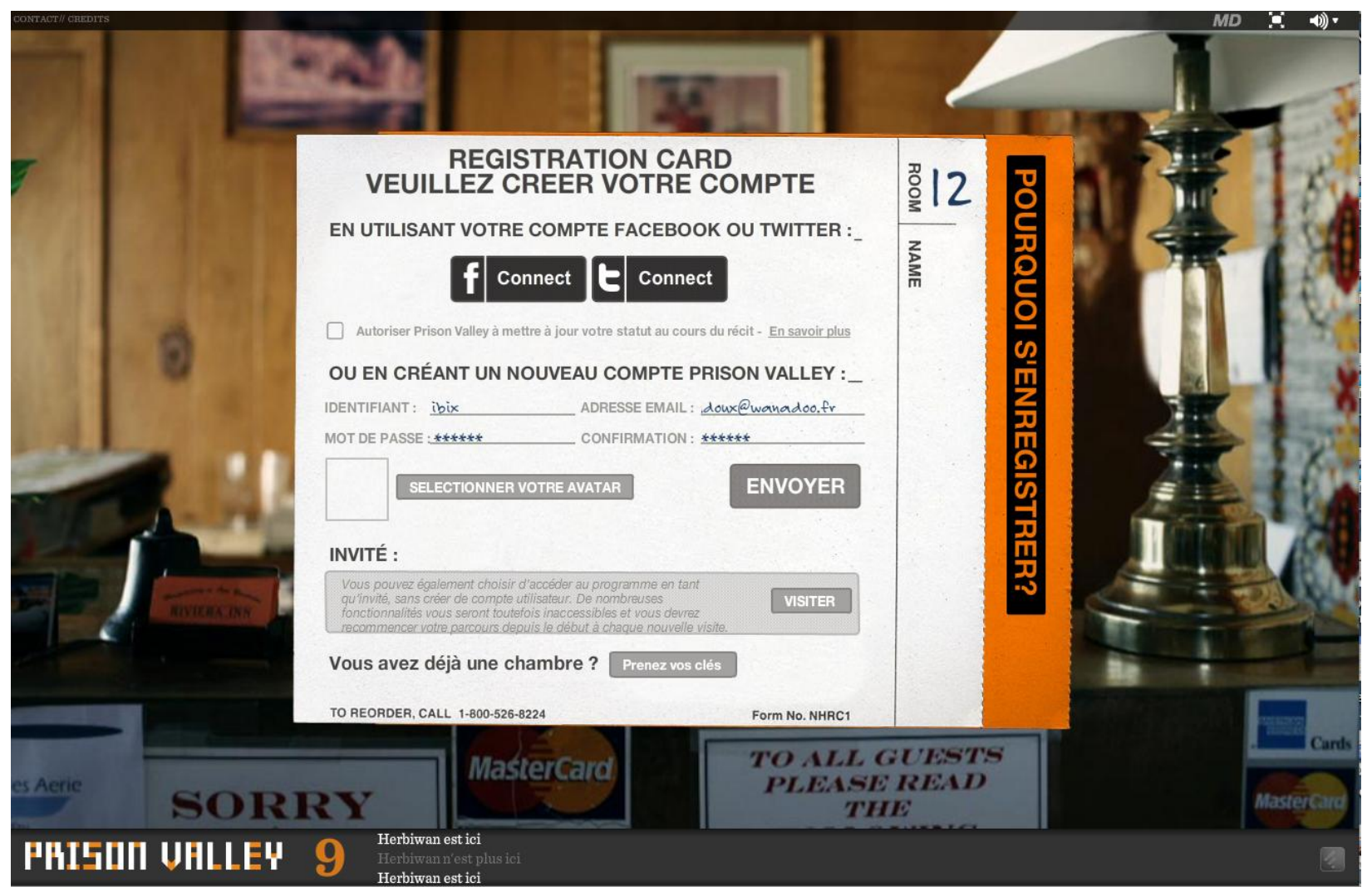

Figure 5 - La « carte d'enregistrement » du visiteur posée sur la vitre de l'accueil du Motel et la chambre, métaphore de l'espace personnel de l'internaute inscrit.

\section{L'encadrement éditorial et la gestion documentaire}

L'encadrement éditorial du web-documentaire dépend de ses modes de financement et des choix de publication. Si l'œuvre possède son propre nom de domaine comme pour Brèves de trottoir ou Prison Valley, les mentions éditoriales sont intégrées au web-documentaire mais elles s'externalisent dans le paratexte lorsque les œuvres sont organisées en séries comme pour France5 ou le Monde.fr.

Si le dépôt de commentaires des internautes apparaît être un choix indépendant des plateformes éditoriales, remarquons que la connexion avec les réseaux sociaux de type Facebook apparaît inévitable à la notable exception de L'obésité est-elle une fatalité ? qui propose d' " envoyer un message aux auteurs » plutôt que de connecter à un réseau social. Orange intègre IRock dans sa communauté Video Party, comprenant des recommandations Vidéo Buzz, une pause humour, et les vidéos les plus vues directement en lien avec sa thématique musicale.

La dernière superproduction d'Arte, Insitu, qui met en scène des interventions artistiques expérimentales dans différentes villes d'Europe accorde une large place à la participation puisqu'il est demandé à l'internaute de télécharger, à partir d'une carte géolocalisant les objets choisis, des documents (textes, images, films) qu'il juge digne d'intérêt parce qu'entrant en résonnance avec ce qu'il a visionné.

Les parties documentaires peuvent être internes et manipulables en tant qu'indices iconiques d'une histoire (photos de détenus dans Prison Valley), incluses dans le dispositif narratif (bloc-notes de dossiers et d'articles du Challenge) ou éditorial (fiches de IRock). Elles peuvent être externes et se présenter sous forme de liste de liens vers des sources internes à l'éditeur (Le corps incarcéré) ou externes (Happy World). Notons que d'un point de vue sémiotique la part faite au texte est belle : retour en force des 
légendes et dialogues, lexiques, références bibliographiques et bibliographies. Celui-ci appuyant la narration ou bien se situant en arrière-plan, en tant qu'annexes.

Cette épaisseur créée par les différentes couches possibles de documentation accrédite l'idée d'un feuilletage possible des composantes hétérogènes de la réalité.
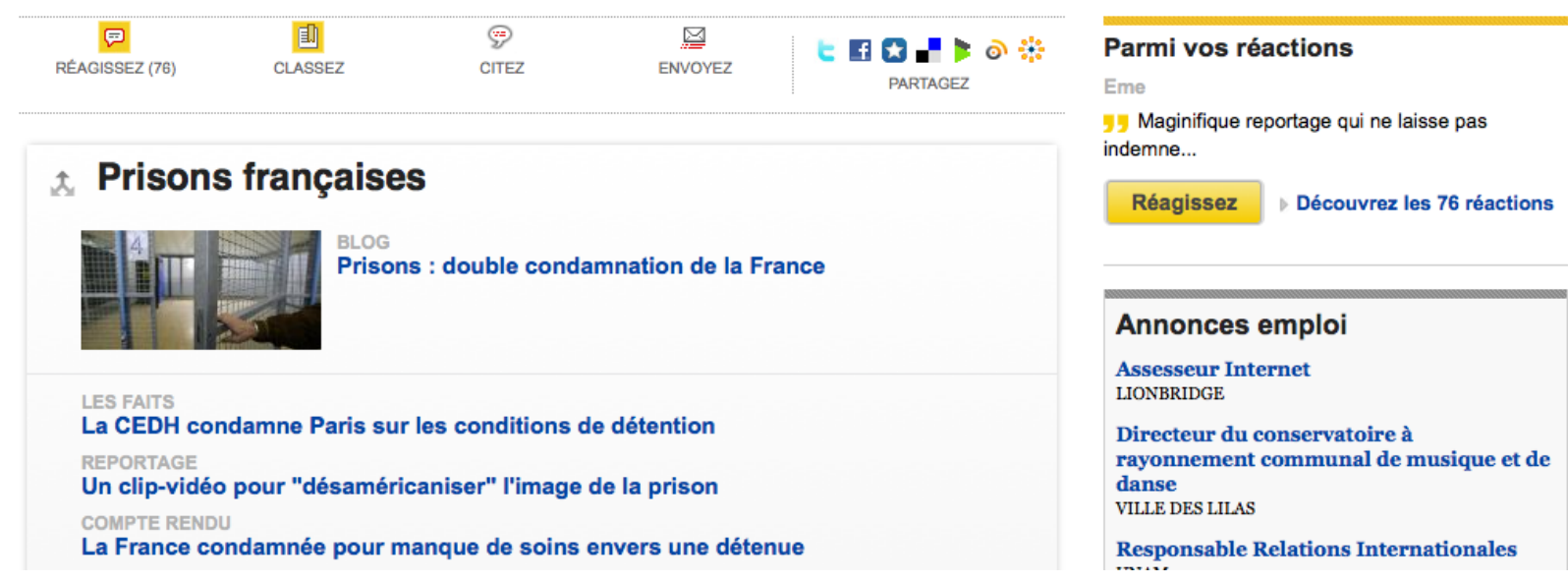

Figure 6 - Sous le Corps incarcéré, on retrouve les incitations classiques du web2.0 à partager, classer et citer mais aussi une connexion avec les contenus du journal en rapport avec la thématique partagée.

\section{L'INTERACTIVITE COMME PRINCIPE D'EXPLORATION ET DE MONTAGE}

\section{La place prise par l'internaute au sein des différents types de web-documentaires}

Au sein des récits à embranchements multiples, l'internaute est amené à jouer différents rôles prévus dès la scénarisation du récit principal ou installés a posteriori. Un lexique employé de manière systématique, caractéristique des «aventures dont vous êtes le héros» interpelle l'internaute: «Vous devez», « Choisissez », «Je reviens », « Tu peux », etc. Cette place déterminante prise par l'internaute indique une modification dans la conception des récits liés à la documentarisation.

L'introduction de L'OBÉSITE EST-ELLE UNE FATALITÉ ? pose les conditions de visualisation du webreportage.

«Vous débutez votre enquête en Seine-Saint-Denis, un des départements les plus touchés de France, 20\% de la population y est obèse ».

« Je cherche à rencontrer des patients »

« J'attends la fin de l'opération pour parler avec le chirurgien »

Bien qu'il s'agisse d'une enquête, l'internaute n'est pas identifié à celui d'un journaliste, mais bien plutôt celui de «protagoniste » convié à découvrir une thématique. Des phrases incitatives comme «Vous avez un léger sentiment d'écœurement » dictent à l'internaute un comportement en prescrivant ses émotions.

Le rôle principal que l'internaute assume dans sa visite exploratoire d'IROCK est celui du « passager clandestin » car la plupart des scènes sont prises sur le vif et l'internaute y assiste en témoin, quelquefois 
même en voyeur puisque les acteurs filmés réagissent souvent de manière émotionnelle à la présence intempestive de la caméra et des micros. Pendant la phase de téléchargement du site, défilent les phrases suivantes.

« Tu va pouvoir explorer les coulisses des eurockéennes »

«18 artistes à suivre»

«A toi de jouer »

Ce tutoiement péremptoire qui donne le « la » indique aussi ce qui est attendu de l'internaute : une forme d'activité liée à l'exploration ludique. Par exemple, la proposition «tu tentes de retrouver un groupe que les journalistes redoutent d'interviewer » est tentatrice et prometteuse car elle repose sur l'imprévu.

C'est dans LE CHALLENGE que l'on trouve la plus grande richesse narrative organisée autour de l'activité de l'internaute. Placé dans la position du journaliste chargé de mener des entretiens, il doit s'organiser et choisir les questions qu'il veut poser :

«Des travaux scientifiques ont-ils été effectués sur la contamination?»

«Quelles sont ces maladies liées à la pollution?»

A chaque fin de séquence, de nouvelles possibilités s'affichent l'obligeant à choisir entre espace narratif et espace documentaire.

« J'étudie les rapports épidémiologiques »

« Je ressors de l'hôpital »

L'internaute est également relancé par des éléments narratifs qui l'immergent dans sa quête et prouvent que son rôle a été scénarisé avant la prise de vues. Par exemple, alors que le juge qui suivait l'affaire a été révoqué et que l'enquête apparaît classée, il reçoit de manière anonyme une enveloppe contenant des documents qui concernent la pollution de la zone naturelle de Yasuni occasionnée par des fuites de pétrole. Le voilà donc reparti pour une série de recherches.

Si l'internaute quitte le web-documentaire et qu'il se reconnecte ultérieurement, il lui est proposé de « recommencer au début » ou de « reprendre l'enquête où je l'ai laissée ». Comme pour PrisonValley, la scénarisation atteint aussi l'entrée/sortie du web-documentaire. 


\section{LE CHALLENGE}

Une enquête interactive de Laetitia Moreau

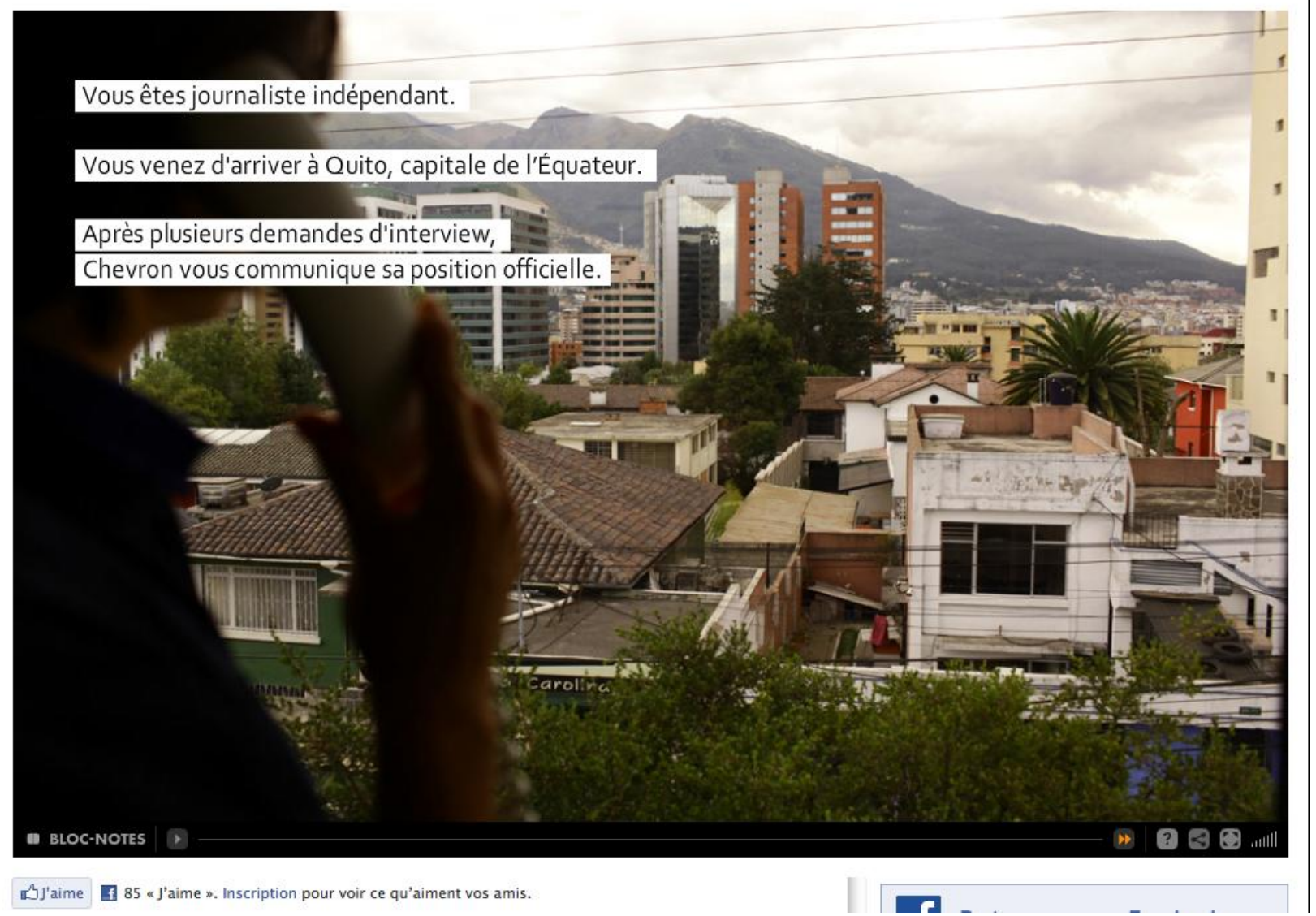

Figure 7 - Représentation physique du lecteur pendant l'exposition de la mission qu'il doit accomplir.

Alors que Pierre Barboza avait établi les allers-retours entre espaces narratifs et documentaires de la bande dessinée interactive "Opération Teddy Bear», les unités intégratives associant les différents espaces discursifs des web-documentaires sous forme de récits à embranchements multiples sont emblématiques d'un renversement. Le lecteur fictionnalisé est entré dans le récit documentaire comme le représente le carton d'enregistrement de Prison Valley sur la figure 5 et la représentation physique de l'interacteur du Challenge sur la figure 6 attribuant à l'internaute une «identité narrative » au sens de Ricoeur.

UBI-SCREEN est un dispositif constitué par l'enregistrement vidéo de conversations que mènent à distance deux interlocuteurs par webcams interposées. La capture est multi-caméras : celle de l'auteur qui filme un protagoniste chez lui pendant que sont enregistrées simultanément les images des webcams des deux participants.

Le résultat pour l'internaute consiste en un écran-type qui comporte un menu en haut de page, une image vidéo qui occupe les trois quarts du milieu de page et d'une ligne temporelle des vidéos enregistrées par phases numérotées. 
Pendant la consultation sur la vidéo principale, un point rose sous forme de cible simplifiée surgit au survol de la souris signalant la possibilité pour l'interacteur de faire apparaitre une seconde image en transparence de la première pouvant être soit une capture du personnage d'un autre point de vue, soit de celui avec lequel il communique par webcam interposée.

L'interacteur alors se trouve dans la situation d'agir sur des images superposées, en mouvement. Mais les images sont brouillées et mobiles et il est difficile de voir nettement l'interlocuteur situé à distance.

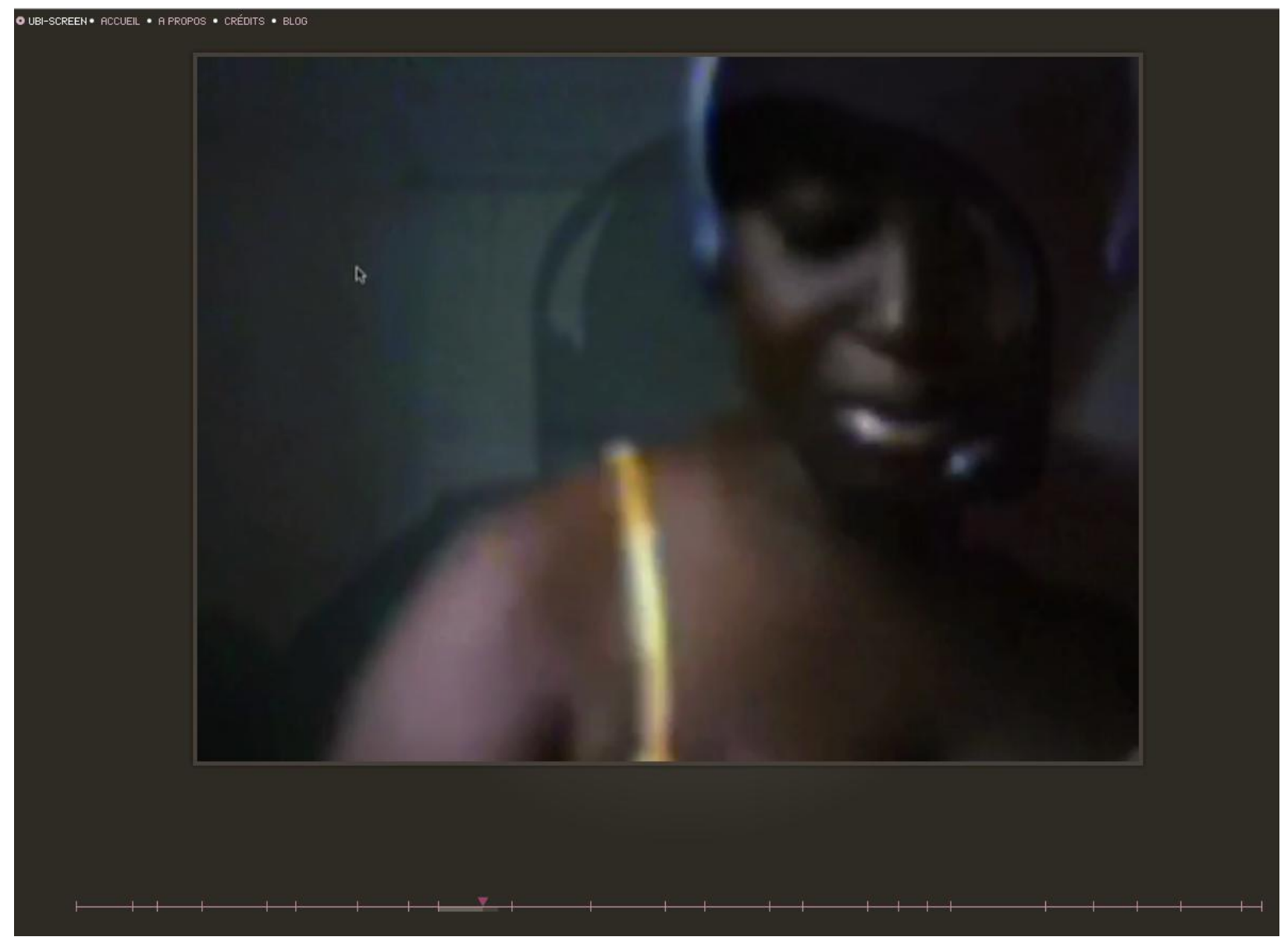

Figure 8 - Apparition furtive d'un protagoniste distant grâce à une action sur image de l'internaute

Ce travail sur l'intime est réalisé par une collaboration entre l'artiste plasticienne Isabelle Grosse et la sociologue Dana Diminescu, spécialiste des usages des nouvelles technologies chez les migrants. Le projet devrait accueillir les témoignages de nouvelles familles dans des situations différentes.

Bien qu'éloignée du documentaire vidéo traditionnel, la captation en temps réel de conversations personnelles par des personnages vivant réellement une séparation est intéressante de par les actions sur images proposées à l'internaute qui se situent dans la rhétorique de l'apparition/disparition décrite par Xavier Malbreil.

«Apparaître/disparaître. »

«Sur la surface blanche de l'écran, ces spectres que l'on peut faire apparaître et disparaître à volonté, ou qui parfois s'imposent à vous, et ne veulent plus s'en aller quand on les a appelés. » 
«La fonction "show/hide layer" comme manière de formuler, en préalable, un questionnement de l'écran. $[\ldots] »$

«Et d'ailleurs (parenthèse), pourquoi tant d'informaticiens s'intéressent-ils à la littérature (ou à l'écriture peut-être davantage ?) ?». ${ }^{9}$

Ici aussi, le lecteur est fictionnalisé. Dans une position de voyeur lorsqu'il consulte simplement les enregistrements vidéo de conversations privées, il passe à une position d'acteur lorsqu'il manipule les images et expérimente la solitude des protagonistes et les flous engendrés par de grandes périodes de temps non partagé. Sa compréhension est facilitée par une métaphore projective.

\section{L'écriture scénaristique conduite par des outils}

Bifurcation des récits et interactivité sont mis à la portée des auteurs, monteurs, réalisateurs et journalistes, qui sont à même d'intégrer différents fichiers sons, images, vidéos dans un logiciel multimédia comme Klynt qui génère au final un « site web » en Flash. La prise en main de l'outil est basé sur la définition préalable d'une arborescence en deux niveaux: le niveau 1 qui représente la "page d'accueil » et ses liens et le niveau 2 qui est celui du montage interactif proposant la possibilité de créer plusieurs parcours à partir de plusieurs entrées. Différentes fonctionnalités permettent de gérer les pistes sons, timeline, scripts, traductions et légendes; la mise en forme graphique étant réalisée par des graphistes et des développeurs au sein de la société Klynt. Les premiers retours d'usages de ce logiciel indiquent un déplacement dans l'écriture du scénario qui peut se faire directement avec l'outil.

De son côté, 3WDOC fait le pari du montage opensource en ligne en profitant des dernières innovations technologiques d'HTML5. Là aussi, sont prédéterminés un ensemble de possibles : ligne temporelle, gabarits, intégration de film, montage de diaporama interactif, menu, carte, etc.

\section{CONCLUSION}

\section{L'entrée du documentaire dans les techniques de narration hypertextuelle}

La partie énonciative du documentaire peut se trouver profondément modifiée par l'usage qui est fait de l'interactivité. Elle entraine l'internaute à endosser les habits de personnages-narrataires pris dans une histoire à laquelle il est sommé d'adhérer (Le challenge) ou bien à se comporter en une sorte de témoinvoyeur (IRock, Ubi-Screen) ou de témoin-enquêteur (Prison Valley, L'obésité). Alors que la voix de l'auteur-narrateur est présente dans Prison Valley, elle disparaît totalement dans Le Challenge. Plus l'immersion de l'internaute est sollicitée dans le récit, moins la nécessité d'un médiateur se fait sentir. C'est pourtant une des caractéristiques du documentaire que d'installer des médiateurs (Happy World) afin d'entraîner le spectateur-auditeur à vivre un récit-témoignage.

Réservée jusqu'alors à l'expérimentation littéraire numérique, le récit hypertexte apparaît faire son entrée dans une sphère qui lui était encore inaccessible il y a peu. L'intérêt des créateurs audiovisuels et photographiques, auteurs et journalistes, pour la publication web laisse augurer de nouveaux essais narratologiques entrainant l'internaute dans des attitudes de narrataires plutôt que de lecteurs. Cependant, cette adhésion au récit ne va-t-elle pas à l'encontre de ce que recherche à l'origine le documentaire ? Que devient la distanciation nécessaire à la réflexion si le contrat de lecture consiste simplement à investir l'internaute dans une « histoire dont il est le héros » entre deux séances de surf sur le web...

\footnotetext{
${ }^{9}$ Message de Xavier Malbreil intitulé Apparition/Disparition à la liste E-critures le 17 juin 2001.
} 
Le web-documentaire est un genre éditorial se déclinant en séries et qui se place au sein du paysage hyperdocumentaire (portail, plateformes, etc.). Les conventions d'écriture repérables sont largement inféodées aux choix techniques de publication sur le web (structure arborescente, ligne temporelle, actions sur images, listes de liens, etc.). Les structures à bifurcations multiples adoptées nécessitent une fragmentation du récit, les interfaces métaphoriques contribuent à installer l'internaute dans la narration en même temps qu'elles règlent les parcours de visionnage et la lecture. Prévue dans le scénario et avant la prise de vues, l'intervention du lecteur internaute participe à la fictionnalisation documentaire.

Redonnant aux témoignages de la vie quotidienne une nouvelle force, les web-documentaires réussissent à faire passer l'épaisseur du réel à travers un récit fragmenté et délinéarisé.

La large panoplie d'acteurs investis, leurs partenariats, la création et le remodelage d'entreprises autour de savoir-faire en formation, la refonte de métiers qui pourrait en découler, sont des indicateurs de l'effervescence créative autour du web-documentaire. Le financement public a donné un coup d'accélérateur à la création. Elargi au web, le documentaire transforme les manières d'informer en offrant aux internautes de nouvelles formes d'expérimentation des réalités décrites.

\section{BIBLIOGRAPHIE}

Barthes, Roland. «L’analyse structurale du récit ». Communications nº 8. Seuil, 1981 (1966).

Gabszewicz Jean, Sonnac Nathalie. «L'industrie des médias à l'ère numérique ». Repères/La Découverte, 2010.

Barboza Pierre. "Fiction interactive, " métarécit 》 et unités intégratives » in L'image actée, Pierre Barboza et Jean-Louis Weissberg (dir.). L'Harmattan, 2006.

Chantepie Philippe, Le Diberder Alain. «Révolution numérique et industries culturelles ». Repères/La Découverte, 2010.

Clément Jean. L'hypertexte de fiction: naissance d'un nouveau genre?, in Vuillemain, A. \& Lenoble, M. (éds). Littérature et informatique. La littérature générée par ordinateur. Arras: Artois Presses Université, pp.63-75, 1995. [en ligne] http://hypermedia.univ-paris8.fr/jean/articles/allc.htm (consultée le 22/07/2011)

Collard Anne-Sophie, "La métaphore dans l'hypermédia comme médiateur de contenus », séminaire sur l'appropriation sociale des nouvelles technologies, IPSI, Tunis, février 2005.

Coutard Nathalie, Houle Michel. «Le documentaire et les plateformes numériques : un écosystème en transformation ». Etude de l'Observatoire du documentaire du Canada. Janvier 2011. [En ligne] http://www.obsdoc.ca/res/pdf/Observ_20110203_Etude.pdf (consultée le 22/07/2011)

Genette G. Figures III, Seuil. 1998 (1972)

Jenkins Henry. «Convergence culture. Where old and new media collide ». New York University Press, 2006

Pédauque Roger. «La redocumentarisation du monde ». Editions Cepadues, 2007.

Ricoeur Paul. « Soi-même comme un autre ». Points-Essais, Editions du Seuil, 1996 (1990).

Salmon Christian, «Storytelling ». La machine à fabriquer des histoires et à formater les esprits. La Découverte, 2008. 
Scopsi Claire (coord.). Dossier «Vidéos en ligne : usages, formats, traitement documentaire » in Documentaliste, sciences de l'information, pp. 26-70. № 4. Décembre 2010.

Thorburn David, Jenkins Henry. "Rethinking media change ». The aesthetics of transition. MIT Press, 2004.

Weissberg Jean-Louis. « Présences à distance ». L'Harmattan, 1999.

\section{Web-documentaires étudiés}

(2009) Le corps incarcéré de Sorin Seelow, Léo Ridet, Eric Dedier, Karim El Hadj, et Bernard Monasterelo produit par Lemonde.fr.

(2009) L'obésité est-elle une fatalité ? de Samuel Bollendorff et Olivia Colo. Coproduction Honkytonk et Curiosphere.tv.

(2009) Brèves de trottoir d'Olivier Lambert et Thomas Salvan. Coproduction France Télévision et Darjeeling.

(2010) Prison Valley de David Dufresne et Philippe Brault. Produit par Upian et co-produit et diffusé par Arte.tv.

(2010) Le challenge. Une enquête interactive de Laetitia Moreau. Production Canal+.

(2010) IRock. Une expérience interactive dans les coulisses des Eurockéennes de Lionel Brouet. Production Orange en partenariat avec France-Inter.

(2010) Cuatro horas de Sébastien Daycard-Heid et Cédric Faimali. Série «Portraits d'un nouveau monde - Vivre ensemble » de France5. Une production Narrative, France Télévisions.

(2010) Ubi-Screen d'Isabelle Grosse. Production Regart.net.

(2011) Happy World. Birmanie : la dictature de l'absurde de Gaël Bordier et Tristan Mendès-France. Une expérience hypervidéo coproduite par Cinquième étage production \& Upian.

(2011) Insitu. Les artistes dans la ville. Un film interactif d'Antoine Viviani. Production Providence et Arte. 